\title{
Grating-based Dipole Antenna Configuration for High Gain Directional Radiation characteristics
}

\author{
Sarin V.P', Jayakrishnan M.P' , C.K Aanandan' ${ }^{2}$, Mohanan Pezholil ${ }^{2}$ and Vasudevan Kesavath ${ }^{2}$
}

\author{
${ }^{1}$ Department of Electronics, Government College Chittur, Palakkad, Kerala, India \\ ${ }^{2}$ Centre for Research in Electromagnetics and Antennas, Cochin University of Science and Technology, India \\ E-mail: sarincrema@gmail.com
}

\begin{abstract}
The experimental and simulation studies of the radiation performance enhancement of a dipole antenna using metal strip grating are presented in this paper. The subwavelength imaging configuration of the metal strip grating is utilized for enhancing the radiation performance of the dipole antenna working in the S-band. The resultant design shows a gain of $9 \mathrm{dBi}$ and a front to back ratio of -23 $\mathrm{dB}$ at resonance. The coupling between electric and magnetic resonances provides the necessary impedance matching performance when the antenna is brought in the vicinity of the grating.
\end{abstract}

\section{Introduction}

Electromagnetic wave propagation through periodic structures is well known to the research community over the last century [1]. A periodic arrangement of metallic strips has been used for achieving variable permittivity values and constitutes a well-known category named as artificial dielectrics. Artificial dielectrics are classified into two categories depending upon the polarization of the incident electromagnetic field with respect to the metal strip orientation [2]. The first category constitutes the phase delay type artificial dielectric in which the polarization of the incident field is lying perpendicular to the strip axis. The unit cell of this structure can be represented by a shunt capacitance in the equivalent circuit diagram and hence it is a low pass prototype. Different configurations of these phase delay type media have been independently studied by W.E Kock and Cohn [3-4]. The delay type materials have been extensively used for the fabrication of microwave lenses. The peculiarity of the lens is that the strip medium achieves permittivity value less than unity and hence a directional radiation pattern can be achieved [5].

The second category is referred as the phase advance type artificial dielectric, in which the polarization of the external electromagnetic field is lying parallel to the axis of the strip. The transmission line model for this type constitutes a shunt inductor and hence it will be a high pass prototype. The dispersion characteristics of this structure shows a close resemblance with that of a rectangular waveguide [2]. This means that we can simulate artificial plasma like environment below the cut-off frequency in a rectangular waveguide [6]. The application of negative permittivity has been used for enhancing the radiation performance of antennas. A negative permittivity material can be represented in the transmission line model by a shunt inductance. This property can be used to match an electrically small electric dipole in order to increase the radiated power [8-9]. An electrically small electric dipole has most of its radiated energy in its capacitive near field which severely affects the radiation performance of the antenna. The surrounding negative permittivity layer gives the necessary inductive reactance and hence impedance matching can be easily obtained. The most common method for getting directional radiation from a dipole antenna is to employ metallic backing. But the main disadvantage is that the image currents induced on the ground plane reduce the radiation efficiency of the antenna [10]. This can be overcome by loading textured High Impedance Surfaces (HIS) on the ground plane. The reflection from the HIS ground plane will be in phase with the incident radiation and hence radiation performance of the antenna is enhanced [1113]. It is interesting to observe that an array of metal rods exhibits a special feature known as canalization in which the half wavelength Fabry-Perot resonant mode is excited yielding subwavelength imaging [14]. The canalization regime can be employed for enhancing the radiation performance of a dipole antenna embedded within the strip medium[15]. Silverihna et. al. achieved non-diffractive infinite directivity beams from a point dipole sources embedded within an array of metal rods [16].

It is well known that an electrically small dipole antenna has a reactive near field and this reactive energy distribution in the near field is a major factor affecting the antenna radiation performance [9]. The near field reactive energy can be perturbed using periodic inclusions so that the evanescent field in the near field can be converted into a propagating far field. Eleftheriades used subwavelength grating structures in order to convert evanescent waves in the near field to far field propagating waves [17]. He also achieved enhanced radiation performance from point source arrays from a metallic grating structure [18]. In this paper, we are proposing the near field imaging configuration used by Tretyakov [19] for enhancing the radiation performance of a half wavelength dipole antenna. In this paper, a single metallic strip grating is utilized to enhance the radiation performance of the antenna. The resultant antenna provides an enhanced gain of about $9 \mathrm{dBi}$ with a front to back ratio of $23 \mathrm{dBi}$. The proposed antenna is analyzed using the Finite 
Element based Ansoft HFSS software and experiments are validated using the PNAE8362B network analyzer. The placement of the dipole antenna near the grating introduces the magnetic resonance and the coupling between the electric and magnetic resonances enhances the impedance matching characteristics of the antenna. The coupling between electric and magnetic resonances also achieves directional radiation performance with improved front to back ratio. Computational analysis based on the Finite Difference Time Domain (FDTD) technique has been performed on the antenna structure to fully understand the resonant mechanism.

\section{Antenna Geometry}

The sketch of the proposed antenna configuration is plotted in fig.1. Fig. 1(a) shows the top view of the antenna. The metal strip grating is printed on a low-cost epoxy substrate of dielectric constant 4.4 and height $\mathrm{h}=1.6 \mathrm{~mm}$. The grating period is denoted by $\mathrm{L}$ and the width of the grating metallization is W. The grating structure occupies an overall area of $255 \times 180 \mathrm{~mm}^{2}$ and utilizes 14 rectangular metal strips in a single array. The dimensions of the grating are selected to be $\mathrm{L}=12.2 \mathrm{~mm}$ and $\mathrm{W}=9 \mathrm{~mm}$. The grating can be called as a subwavelength grating because of the fact that the grating period (L) is lesser than the wavelength of operation in the S-band. Hence the diffraction orders will be absent and the grating will be opaque to incident electromagnetic radiation for TE polarization. If a dipole antenna is placed in the vicinity of the grating, the reflected waves from the grating will naturally deteriorate the radiation efficiency of the antenna. But it is observed that the placement of the dipole near the grating introduces the magnetic resonance. The electric and magnetic resonances of the composite system can be merged and hence impedance matching and radiation performance of the antenna can be improved. Fig. 1(b) shows the loaded dipole antenna configuration. The printed dipole antenna, fabricated on the same epoxy substrate, has a dimension of

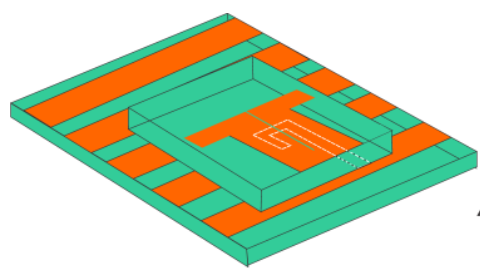

(a)

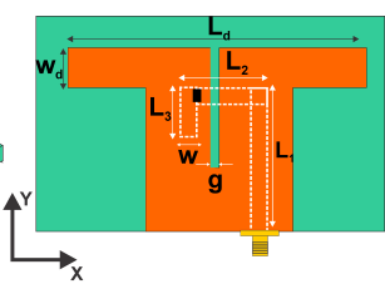

(b)

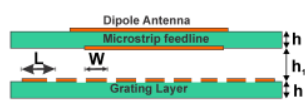

(c)

Figure 1: Geometry of the antenna a) Top view, b) Dipole antenna configuration and c) side view

$\mathrm{L}_{\mathrm{d}}=34.5 \mathrm{~mm}, \mathrm{~W}_{\mathrm{d}}=5 \mathrm{~mm}$ and the feed gap $\mathrm{g}=0.5 \mathrm{~mm}$. The dipole antenna is fed using a standard microstrip to slot line transition. The dimensions of the balun are $\mathrm{L}_{1}=18 \mathrm{~mm}$, $\mathrm{L}_{2}=10 \mathrm{~mm}, \mathrm{~L}_{3}=8 \mathrm{~mm}$ and $\mathrm{W}_{\mathrm{m}}=3 \mathrm{~mm}$. The microwave signal is fed using the Sub Miniaturized Version A (SMA) connector soldered at one end of the microstrip line which is subsequently connected to the coaxial cable port of the network analyzer. The dipole antenna is loaded at a height of $h_{1}=10 \mathrm{~mm}$ from the grating and is shown in the side view illustrated in fig.1(c). The optimum loading height is determined after running rigorous parametric optimization in Ansoft HFSS. For experimental realization, the dipole antenna is suspended using Perspex spacers located at its four corners. The grating and the antenna are fabricated using standard photolithographic procedures.

\section{Results and discussions}

Initially, the effect of stacking height $\mathrm{h}_{1}$ on the reflection characteristics of the antenna is studied. For that, the parameter $h_{1}$ is varied from $2 \mathrm{~mm}$ to $14 \mathrm{~mm}$ and the corresponding reflection characteristics are plotted in fig. 2 . It can be seen that for $h_{1}=2 \mathrm{~mm}$, two unmatched resonances are observed at $2.87 \mathrm{GHz}$ and $4.21 \mathrm{GHz}$. The reflection coefficients at these resonances are found to be $-2.53 \mathrm{~dB}$ and $-4.4 \mathrm{~dB}$ respectively. As the stacking height is increased, the lower resonance shows better impedance matching due to the reduction in capacitive coupling between the antenna and the strip grating. This resonance is identified as the electric resonance of the dipole antenna. The higher

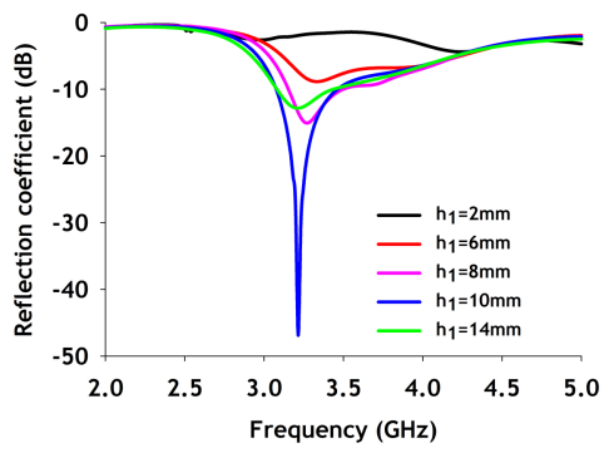

Figure 2. Effect of loading height $h_{1}$ on reflection Coefficient

resonance is showing a lower shift with an increase in stacking height and it denotes the magnetic resonance of the composite. The magnetic resonance is contributed due to the anti-symmetric currents on the plates constituting a circular current loop between the dipole and the grating. Optimum stacking height denotes the one in which the electric and magnetic resonances merges with each other $\left(\mathrm{h}_{1}=10 \mathrm{~mm}\right)$ and shows the best impedance matching performance. The resonant frequency is found to be $3.21 \mathrm{GHz}$ at the optimum loading height and the corresponding reflection coefficient value at resonance is found to be $-47 \mathrm{~dB}$. Increasing the loading height further decreases the impedance matching performance of the antenna. It is to be noted that the electric resonance creates electric polarizability and the magnetic resonance creates magnetic polarizability. Here, the coupling between electric and magnetic resonance contributes better impedance matching and radiation performance for the dipole antenna. 
In order to understand the resonant behavior further, the reflection phase at the feed point of the antenna is observed for each variation in stacking height. The corresponding variation in reflection phase at the input port with the loading height $h_{1}$ is shown in fig. 3. It is observed that for lower stacking heights $\left(h_{1}=6 \mathrm{~mm}\right.$ and $\left.h_{1}=8 \mathrm{~m}\right)$, the

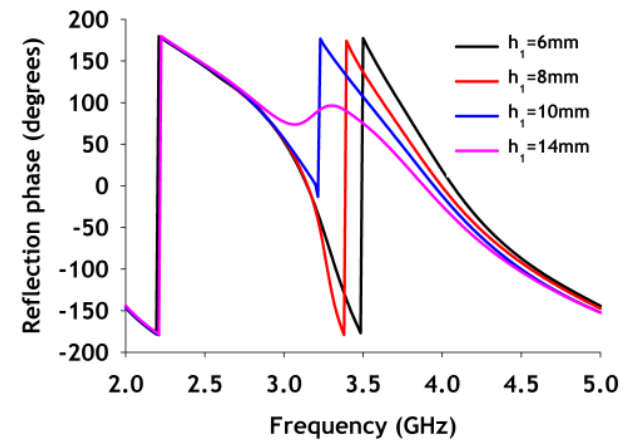

Figure 3. Effect of loading height $h_{1}$ on reflection phase at the input port

reflection phase will be highly capacitive and hence impedance matching performance will be deteriorated for the lower resonance. The change in reflection phase with frequency is negative for both these cases and hence impedance matching and bandwidth of the design will be poor. For the optimum stacking height of $h_{1}=10 \mathrm{~mm}$, the reflection phase is reduced to zero at the resonant frequency, indicating the merging of electric and magnetic resonances. The abrupt positive reflection phase gradient with frequency at resonance yields the necessary impedance matching performance for the antenna. It is to be noted that the total reflection phase offered by the grating and by the cavity resonance (magnetic resonance) are in phase which gives the necessary positive phase gradient. Variation in loading height further increases the reflection phase to positive values and the antenna will be mismatched again due to the high inductive reactance.

Parametric studies have been performed on the width $\mathrm{W}$ of the grating metallization on the reflection and radiation characteristics of the antenna. Simulation studies have been performed while keeping the grating period $\mathrm{L}$ as a constant $(\mathrm{L}=12.2 \mathrm{~mm})$ and the results are summarized in fig. 4. It is observed that decreasing the value of strip width severely affects the impedance matching performance of the antenna. A wider strip is required for better impedance matching. Fig. 4(b) shows the effect of variation in reflection phase at the feed point of the antenna with grating strip width. It is seen that for the wider strip ( $W=9 \mathrm{~mm})$, the reflection phase approaches zero, indicating merging of electric and magnetic resonant modes. Decreasing the value of $\mathrm{W}$ shifts the reflection phase to inductive values and hence the impedance matching performance is deteriorated. The reflection phase shows an abrupt positive phase jump for the wider strip width $(\mathrm{W}=9 \mathrm{~mm})$. The positive reflection phase transition is gradual for lower strip widths and the slope of the reflection phase is decreasing as we decrease the metal strip width. The effect of variation in the normalized gain patterns of the antenna with strip width is illustrated in fig. 4(c) and (d). It is observed that the radiated gain is not showing an appreciable change with the change in strip width. For smaller strip widths, the back radiated power of the antenna is increased, resulting in a reduced front to back ratio. The increased back radiated power is attributed due to the leakage through the slits.
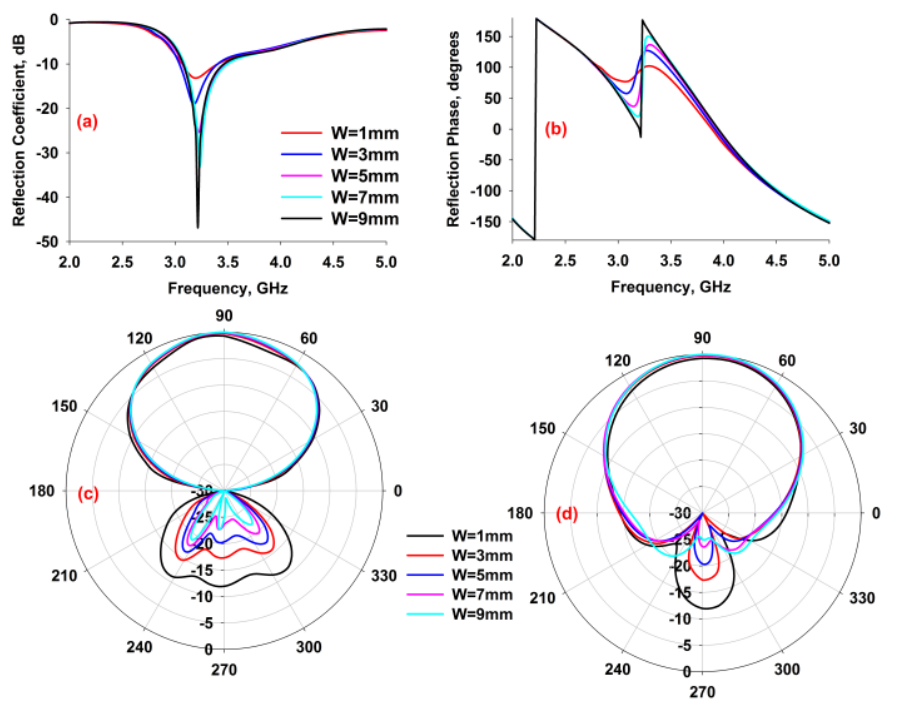

Figure 4. Effect of variation in grating thickness $W$ on reflection and radiation characteristics a) variation in reflection coefficient, b)variation in reflection phase, c) variation in $E$ plane gain pattern and d) variation in $H$ plane gain pattern

The output spectrum of the metal strip grating can be represented as [17],

$$
\mathrm{E}_{\text {out }}\left(k_{x}\right)=\sum_{n=-\infty}^{+\infty} \mathrm{E}_{\text {in }}\left(\mathrm{k}_{\mathrm{x}}-\frac{2 \pi \mathrm{n}}{\mathrm{L}}\right) \mathrm{B}_{\mathrm{n}}\left(\mathrm{k}_{\mathrm{x}}-\frac{2 \pi \mathrm{n}}{\mathrm{L}}\right)
$$

Where $E_{i n}$ is the input spectrum of the incident wave, $B_{n}$ is the weighting function dependent upon the geometry of the grating and $\mathrm{L}$ is the period of the grating. It states that the output spectrum is the weighted copies of the input spectrum shifted by integer multiples of the grating wave number $2 \pi / \mathrm{L}$. Since most of the radiated energy of the halfwavelength dipole antenna is contained in its real low spatial frequency components, the contribution due to the high spatial frequency components is negligibly small. In order to have a negligible spatial shift, the grating period L should have a high value. Therefore the variation in grating thickness $\mathrm{W}$ while making the grating period $\mathrm{L}$ as a constant will not give enhancement in the gain of the antenna. Decreasing the value of grating period shifts the evanescent high spatial components, which is negligibly small as compared to the propagating spectrum and hence the radiated power gain of the antenna remains the same.

Simulation studies have also been performed by varying the period $\mathrm{L}$ of the grating. The effect of variation in reflection characteristics and the normalized gain patterns against the change in grating period is illustrated in fig. 5 . 
Parameter variations are performed by fixing the loading height of the antenna at $\mathrm{h}_{1}=10 \mathrm{~mm}$. It is seen that the reflection coefficient remains unaltered by the corresponding change in grating period. The variation in the normalized gain patterns shows that the radiated power of the antenna remains the same for all the variations. This is due to the fact that in the case of a half wavelength dipole antenna, almost all the energy is contained in its low spatial frequency components and the contribution from the high spatial frequencies are significantly small so that the spatial frequency shift can be neglected. But for an electrically small radiator, all the energy will be contained in its reactive near field and hence the reduction in grating period will cause a significant spatial shift towards the lower side of the spatial spectrum.

A comparison study has been carried out on the performance of the grating backed dipole and that of a typical conductor backed dipole antenna. The metal conductor backing occupies the same area as that of the

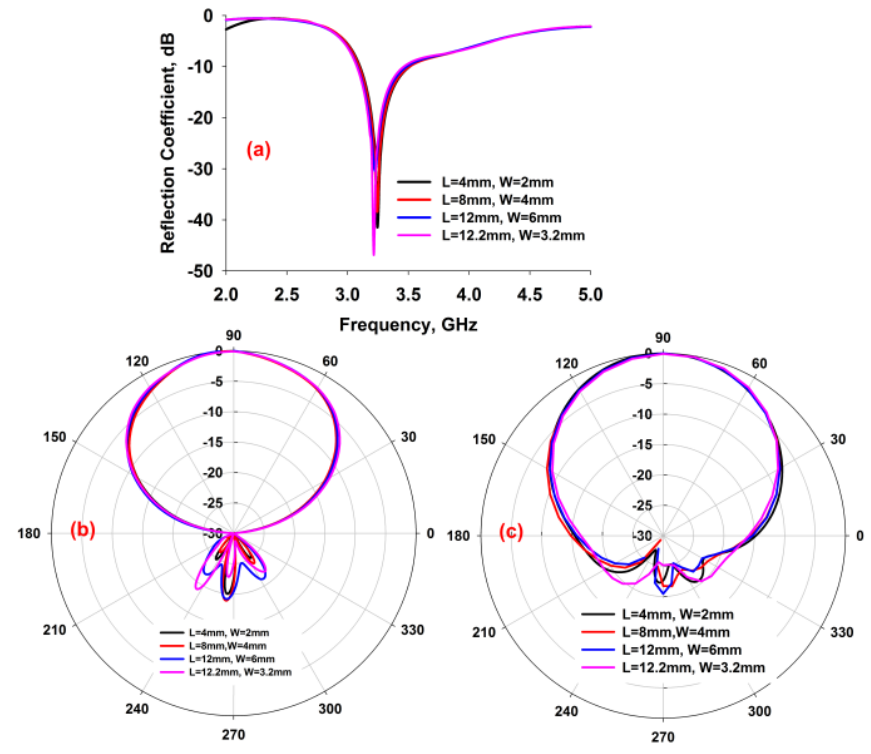

Figure 5. Effect of variation in grating period $L$ on reflection and radiation characteristics a) variation in reflection coefficient, b) variation in E plane gain pattern and d) variation in $H$ plane gain pattern

grating structure $\left(255 \mathrm{X} 180 \mathrm{~mm}^{2}\right)$ and the same loading height $\left(\mathrm{h}_{1}=10 \mathrm{~mm}\right)$ is used for suspension. The resultant reflection coefficient and reflection phase are plotted in fig. 6. It is clearly seen that the conductor-backed dipole is not perfectly matched and the reflection coefficient value is found to be $-8.2 \mathrm{~dB}$ at resonance. A conductor backed dipole antenna requires a loading height of the order of a quarter wavelengths in order to have better radiation efficiency [20].
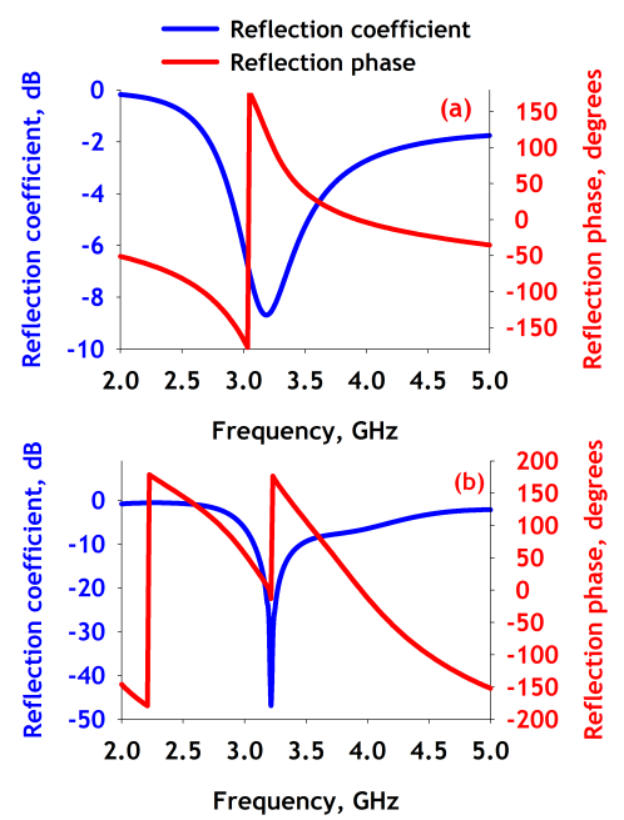

Figure 6. A comparison between the conductor backed dipole antenna and the grating backed dipole a) Reflection coefficient and phase of the conductor backed design and b) reflection coefficient and phase of grating backed antenna

The grating backed design utilizes the coupling between electric and magnetic resonances to get better impedance matching performance compared to the conventional conductor backed configuration. The loading height is of the order of $0.11 \lambda_{0}$ corresponding to the resonant frequency. Another important difference is the reflection phase at the input port of the two designs. The conductor backed design shows negative reflection phase gradient at the resonant frequency, which limits the radiation performance of the design. The grating backed design shows a positive reflection phase gradient and hence the impedance matching and radiation performance will be superior as compared to the conductor-backed design. The positive reflection phase gradient in band gap structures has been used for achieving directional radiation characteristics from dipole antennas [21]. Nowadays, artificial magnetic conductor (AMC) inclusions have been used extensively for increasing the gain of microstrip and dipole antennas [11-12]. The AMC structures require shorting pins to be connected between the upper metal patch and the lower ground plane. In other groundless configurations of AMC structures, more 3D magnetic inclusions are needed to be stacked which increases the fabrication complexities [12]. The advantage of the current design is that the grating structure utilized here is fully planar and only the loading height of the dipole is to be optimized for getting good impedance matching characteristics.

In order to find the resonant behavior of the grating backed antenna, the antenna geometry under consideration is computationally analyzed using the Matlab based Finite Difference Time Domain codes. In FDTD analysis, Maxwell's curl equations are converted into difference 
equations and subsequently solved depending upon the boundary conditions [22]. For that, the computational domain is divided into Yee cells with discretisation factors $\Delta x=\Delta y=0.5 \mathrm{~mm}$ and $\Delta \mathrm{z}=0.4 \mathrm{~mm}$. The resultant computed electric and magnetic field distributions on the dipole antenna structure with and without the metal strip grating are summarized in fig. 7. It can be seen that the grating backed dipole shows the presence of magnetic resonance at the resonant frequency. The $\mathrm{H}_{\mathrm{y}}$ component of the magnetic field distribution shows a cosine-like distribution on both the plates symmetrically. This is due to the anti-symmetric loop current distributions circulating between the antenna plates and the grating. Maximum magnetic field magnitude is observed at the centre of each plate and the minimum values are located quarter wavelength apart on the two sides of the plates. The $\mathrm{E}_{\mathrm{z}}$ component shows maximum amplitude where the magnetic field amplitude is minimum. This type of field distribution is similar to that of a stacked plate dipole antenna configuration and it confirms the presence of magnetic resonance [23]. For an ordinary plate dipole antenna, electric resonance is observed as indicated in Fig 7(a) and (b) and the magnetic resonance will be absent.

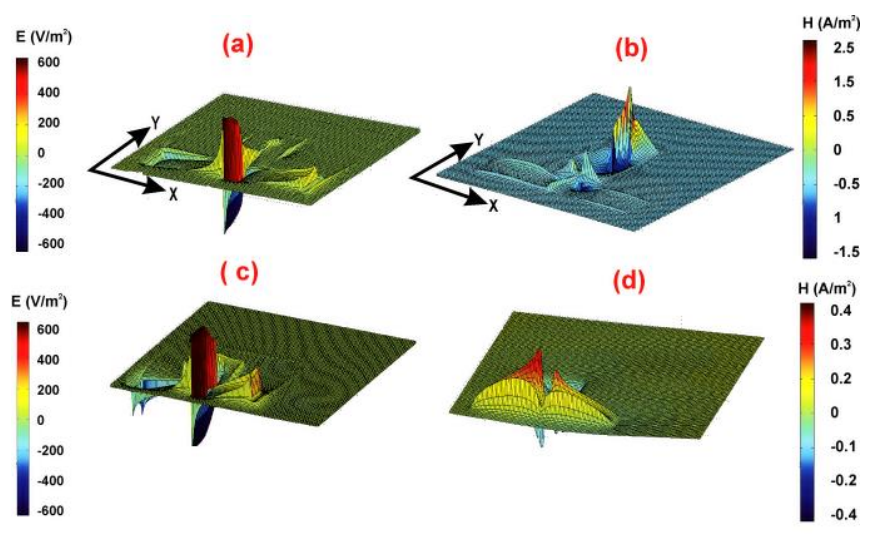

Figure 7. Computed electric and magnetic field distributions of the dipole antenna and grating backed dipole antenna a) $E_{z}$ component of electric field of simple dipole b) $H_{y}$ component of magnetic field of a dipole, c) $E_{z}$ component of electric field of grating backed dipole and $d$ ) $H_{y}$ component of magnetic field of grating backed dipole

The fabricated antenna is tested in an anechoic chamber and the resultant resonant and radiation characteristics for the optimum loading height of $h_{1}=10 \mathrm{~mm}$ are summarized in fig. 8 . Fig. 8 (a) shows the reflection characteristics of the fabricated antenna. The simulation and experiment are showing fairly good agreement with each other. The resonant frequency is found to be $3.25 \mathrm{GHz}$ and the reflection coefficient value is found to be $-22 \mathrm{~dB}$ at resonance. The 2:1 VSWR bandwidth is found to be $9.8 \%$ around the resonant frequency. The measured radiation patterns of the antenna at the resonant frequency for the two principal planes are plotted in fig. 8 (b) and (c). It is observed that the antenna attains directional radiation characteristics with radiation directed along the broadside direction. The antenna shows relatively high cross polar isolation of $-23 \mathrm{~dB}$ in the E-plane and $-20 \mathrm{~dB}$ in the H-plane. The $3 \mathrm{~dB}$ beam width is found to be $65^{\circ}$ in the $\mathrm{H}$ plane and $60^{\circ}$ in the $\mathrm{E}$ plane. The polarization of the antenna is directed along the axis of the metal strip grating. The gain of the antenna is measured using gain comparison method. The measured gain is found to be $9 \mathrm{dBi}$ at resonance at the optimum stacking height. The radiation efficiency, measured using Wheeler cap method, is found to be $90 \%$ at resonance.

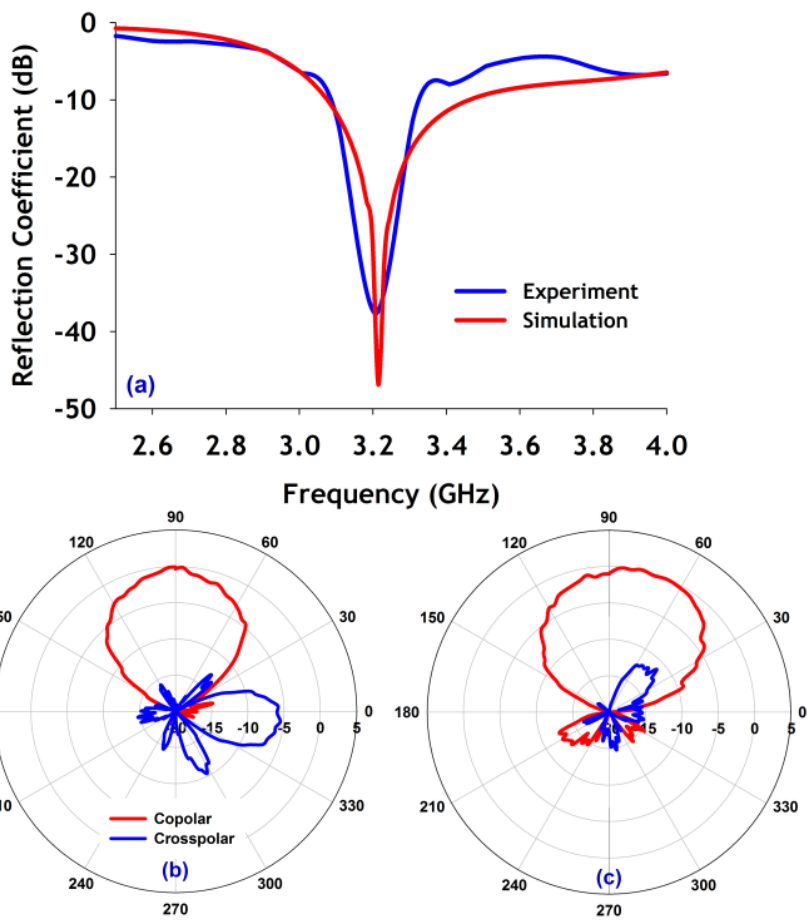

Figure 8. Reflection and radiation characteristics of the fabricated antenna a) Reflection coefficient, b) E plane radiation patterns and c) $H$-plane radiation patterns

\section{Conclusions}

The radiation performance enhancement of a dipole antenna by utilizing a single layer metal strip grating is presented in this paper. The antenna shows better impedance matching performance and the gain is enhanced up to $9 \mathrm{dBi}$. The coupling between electric and magnetic resonance provides the necessary impedance matching performance and gives positive reflection phase gradient as compared to a conventional conductor backed configurations.

\section{Acknowledgements}

This work was supported in part by the University Grants Commission (UGC), Government of India and Department of Science and Technology (DST), Government of India. 


\section{References}

[1] A.F Harvey, "Periodic and guiding structures at microwave frequencies," IRE Tans. Microw. Theory and Tech., vol. 47, no. 10, pp. 30-61, 1960

[2] R.E Collin, "Field theory of guided waves," Second edition, IEEE Press, New York.

[3] Winston E. Kock, "Metal-Lens Antennas," Proc. of IRE and waves and electrons, vol. 47, pp. 2075-2081, 1946.

[4] S.B Cohn, "Analysis of the metal strip delay dielectrics", Journal of Applied Physics, no.20, 1949.

[5] I.H Bahl, K.C Gupta, "A leaky wave antenna using an artificial dielectric medium," IEEE Trans. Antennas and Propag., pp.119-122, 1974.

[6] Walter Rotman "Plasma simulation by artificial dielectrics and parallel plate media," IRE Trans. Antennas and Propag., Vol. 73, pp.92-95, 1962.

[7] J.B Pendry, A.J Holden, W.J Stewart, I. Youngs, "Extremely low frequency plasmons in metallic meso-structures," Physical Rev. Lett., vol. 76, no. 25, pp. 4773-4776, 1996

[8] Richard W. Ziolkowski, Allison D. Kripple, "Reciprocity between the effects of resonant scattering and enhanced radiated power by electrically small antennas in the presence of nested metamaterial shells “, Phys. Rev. E, vol. 72, pp. 036602(1-5), 2005.

[9] Richard W. Ziolkowski, Allison D. Kripple, "Application of double negative materials to increase the power radiated by electrically small antennas ", IEEE Trans. Antennas and Propag., vol. 51, no. 10, pp. 2626-26402003.

[10] A. Foroozesh and L. Shafai, "Application of combined electric- and magnetic-conductor ground planes for antenna performance enhancement" Can. Journal of Elect. Comp. Eng., vol. 33, no. 2, pp. 87-98, 2008

[11] Dan Sievenpiper, Jim Schaffner, J.J Lee, Stan Livingston, "A steerable leaky wave antenna using a tunable impedance ground plane", IEEE Antennas and wireless propag. Lett, vol. 1, pp.179-182, 2002.

[12] Sarin V.P, Jayakrishnan M.P, Aanandan C.K, Pezholil M, Kesavath $\mathrm{V}$, "A Metamaterial backed dipole antenna for high gain directional communications", Advanced Electromagnetics, vol. 5, no. 1, 2016.

[13] Aycan Erentok, Paul L. Luljack, Richard W. Ziolkowski, "Characterization of a volumetric metamaterial realization of an artificial magnetic conductor for antenna applications", IEEE Trans. Antennas and Propag. vol. 53, no. 1, pp. 160-172, 2005.

[14] P. A. Belov, C. R. Simovski, "Canalization of subwavelength images by electromagnetic crystals," Physical Rev. B, vol. 71, no. 193105, pp. $1-4,2005$.

[15] S. Kosulnikov, D. Filonov, S. Glybovski, P. Belov, S. Tretyakov C. Simovski, "Wire-Medium Hyperlens for Enhancing Radiation From Subwavelength Dipole Sources," IEEE Trans. Antennas and Propag. vol. 63, no. 11, pp. 4848-4856, 2015.

[16] T. A. Morgado and M. G. Silveirinha, "Super-collimation of the radiation by a point source in a uniaxial wire medium," EPJ Appl. Metamat., vol. 2, no.14, pp. 2-8, 2016.

[17] Mohammad Memarian, George V. Eleftheriades, "Evanescent to propagating wave conversion in subwavelength metal strip gratings", IEEE Transactions on Microwave Theory and Techniques, 60 (2012), 3893-3907.

[18] Mohammad Memarian, George V. Eleftheriades, "Enhanced radiation of an invisible array of sources through a sub-wavelength metal-strip grating and applications", Journal of Applied Physics, 114 (2013), 134902.

[19] G. Fedorov, S.I Maslowski, A.V Dorofeenko, A.P Vinogradov, I.A Ryzhikov, S.A Tretyakov, "Subwavelength imaging: Resolution enhancement using metal wire gratings", Physical Review B, 73 (2006), 035409 (1-7).

[20] A. Foroozesh and L. Shafai, "Application of combined electric and magnetic conductor ground planes for antenna performance enhancement", Canadian Journal of Electrical and computer engineering, 33 (2008), 87-98.

[21] Yuehe Ge, Karu P. Esselle, Trevor S. Bird, "The use of simple thin partially reflective surfaces with positive reflection phase gradients to design wideband, low profile, EBG resonator antennas", IEEE Transactions on Antennas and Propagation, 60 (2011), 743-750.
[22] D. M. Sullivan, "Electromagnetic Simulation Using the FDTD Method," IEEE Press series on RF and microwave technology, 2000.

[23] Sarin V. Pushpakaran, Nishamol M. SeidMuhammed, Rohith K. Raj, Anju Pradeep, P. Mohanan, Kesavath Vasudevan, "A compact stacked dipole antenna with directional radiation coverage for wireless applications", IEEE Antennas and Wireless Propag. Lett., vol.12, pp. 841-844, 2013 